\title{
Time-dependent greenhouse warming computations with a coupled ocean-atmosphere model
}

\author{
Ulrich Cubasch ${ }^{1}$, Klaus Hasselmann ${ }^{2}$, Heinke Höck ${ }^{2}$, Ernst Maier-Reimer ${ }^{2}$, Uwe Mikolajewicz ${ }^{2}$, \\ Benjamin D Santer ${ }^{2}$, and Robert Sausen ${ }^{3}$ \\ ${ }^{1}$ DKRZ, Bundesstrasse 55, D-20146 Hamburg, Germany \\ ${ }^{2}$ Max-Planck-Institut für Meteorologie, Bundesstrasse 55, D-20146 Hamburg, Germany \\ ${ }^{3}$ DLR, Institut für Physik der Atmosphäre, D-82234 Oberpfaffenhofen, Germany \\ Climate Dynamics (1992) 8:55-69
}

The color bar and the associated contour interval identification were inadvertently omitted from Fig. 12 of the above article. The color bar and the revised figure caption are printed below.

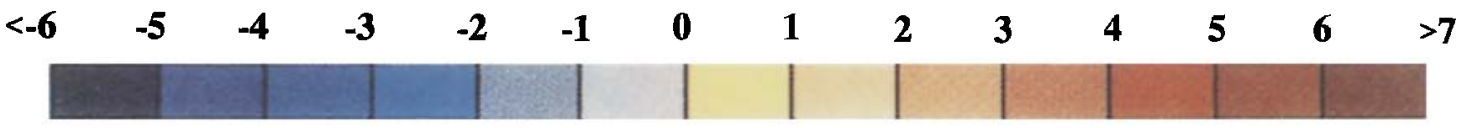

Fig. 12.a Distribution of the change in annually-averaged near surface temperature for the final decade (years 91-100) of the Scenario A integration relative to the initial state (averaged over years 1-10) of the control run (contour interval: $1 \mathrm{~K}$ ); b EOF 1 of the annually-averaged near surface temperature changes for Scenario A. The explained variance is $84.1 \%$ (contour interval:

$1 \times 10^{-2}$ ); c EOF 2 of the annually-averaged near surface temperature changes for Scenario $\mathrm{A}$. The explained variance is $3.1 \%$ (contour interval: $1 \times 10^{-2}$ ); d EOF 1 of the annually-averaged near surface temperature changes for the control run. The explained variance is $52.5 \%$ (contour interval: $1 \times 10^{-2}$ ) 